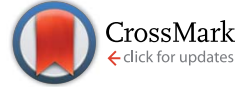

Cite this: RSC Adv., 2014, 4, 31507

Received 11th April 2014

Accepted 23rd June 2014

DOI: $10.1039 / c 4 r a 03282 a$

www.rsc.org/advances

\section{Uniform core-shell molecularly imprinted polymers: a correlation study between shell thickness and binding capacity $\dagger$}

\author{
Zhong Zhang, ${ }^{\text {ab }}$ Lingxin Chen, ${ }^{\text {*a }}$ Fangfang Yang ${ }^{a}$ and Jinhua $\mathrm{Li}^{\text {*a }}$
}

\begin{abstract}
Core-shell molecularly imprinted polymers (CS-MIPs) have aroused increasing interest owing to their easy accessibility and favorable mass transfer. Herein, we explore the correlation between shell thickness and binding capacity by using Sudan I as template molecule to prepare different CS-MIPs at the surface of carboxyl polystyrene through emulsion polymerization with a two-step temperature-rising process. Extensive characterization was performed using techniques such as SEM/TEM, FT-IR, BET, and TGA. Main factors were systematically studied such as the amount of prepolymer solution, the amount of SDS, and the temperature step. Under the optimized conditions, CS-MIPs with a shell thickness of $2.60 \mu \mathrm{m}$ presented the highest binding capacity of $30.1 \mu \mathrm{mol} \mathrm{g}{ }^{-1}$ and the most rapid mass transfer rate. A uniform sphere model was constructed, and it was found that template molecules located in the spherical MIPs with a diameter of $5.20 \mu \mathrm{m}$ will be completely eluted, thereby attaining the maximum binding capacity. The static adsorption isotherm followed the Langmuir-Freundlich adsorption model, and the fast kinetics obeyed the pseudo-second-order kinetics model. High recognition specificity for Sudan I with respect to its analogues was displayed, with an imprinting factor of 2.7. The establishment of a critical value of shell thickness provides new insights into the preparation methodology and molecular recognition mechanism of core-shell imprinted polymers.
\end{abstract}

\section{Introduction}

Molecular imprinting has been widely recognized as a promising technology for the preparation of tailor-made synthetic materials that are capable of specifically recognizing targeted molecules. ${ }^{1}$ For the past few decades, the interest and attention shown toward this technology has been increasing at an amazing pace. ${ }^{\mathbf{1 - 4}}$ This is mainly attributed to the diversity of potential applications of the versatile molecularly imprinted polymers (MIPs) in purification/separation, ${ }^{4,5}$ chemo/biosensing, ${ }^{6}$ catalysis, ${ }^{7,8}$ drug delivery ${ }^{9,10}$ and so on. MIPs exhibit many attractive characteristics; however, they still have met with some limitations, such as incomplete template removal, low binding capacity and slow mass transfer.,11 Considering the above problems, a variety of approaches have been developed, e.g., precipitation polymerization, surface imprinting, controlled/ living free radical polymerization, etc. ${ }^{1}$ Surface imprinting has been accepted as one of the most promising ways, with the

${ }^{a}$ Key Laboratory of Coastal Environmental Processes and Ecological Remediation, Yantai Institute of Coastal Zone Research, Chinese Academy of Sciences, Yantai 264003, China. E-mail: lxchen@yic.ac.cn; jhli@yic.ac.cn; Fax: +86 535 2109130; Tel: +865352109130

${ }^{b}$ University of Chinese Academy of Sciences, Beijing 100049, China

$\dagger$ Electronic supplementary information (ESI) available. See DOI: $10.1039 / \mathrm{c} 4 \mathrm{ra03282a}$ imprinted sites situated at or close to the surface of MIPs, enabling an easy access of the target molecules and fast mass transfer. ${ }^{12}$ Surface imprinting has been extensively studied for many support materials such as silica nanoparticles, ${ }^{\mathbf{1 3}}$ silica gel, $\mathrm{Fe}_{3} \mathrm{O}_{4}$ magnetic particles, ${ }^{14}$ and polystyrene beads ${ }^{\mathbf{1 5}}$ for the formation of surface coatings. Recently, surface imprinting core-shell MIPs (CS-MIPs) have been increasingly synthesized and more widely applied due to their intrinsic advantages, such as good dispersion, rapid binding kinetics, and easy and complete removal of template molecules. ${ }^{13-17}$

In order to satisfy different applications, CS-MIPs with well controlled physical forms in different size ranges are highly desirable. For example, MIP nanoparticles are very suitable in binding assays and microfluidic separations, whereas MIP beads with diameters of 1.5-3 $\mu \mathrm{m}$ are typically more appropriate to use in new analytical liquid chromatography systems. Ye et al. have systematically investigated how to control particle size for different analytical applications and synthesized monodisperse MIP beads with sizes ranging from $100 \mathrm{~nm}$ to 2.4 $\mu \mathrm{m}$ by varying the ratio of two different cross-linkers in precipitation polymerization. ${ }^{18}$ Lu et al. have used a modified precipitation polymerization method to synthesize microspheres with diameters of about 2-3 $\mu \mathrm{m} .{ }^{19}$ The mechanism and influence factors of this polymerization method were studied. The research on shell thickness for binding capacity, however, is quite limited..$^{20}$ One example was presented by Zhang et al., 
where they developed a surface functional monomer-directing strategy at the surface of silica nanoparticles. ${ }^{20} \mathrm{~A}$ critical value of shell thickness for the maximum rebinding capacity was determined by testing the evolution of rebinding capacity with shell thickness, which provided insight into the effectiveness of molecular imprinting and the form of imprinted materials. Therefore, investigating the relationship of shell thickness and the imprinting performances of CS-MIPs may offer important guiding significance to attaining ideal CS-MIPs.

In this work, we explore the influence of shell thickness on CS-MIP properties, by preparing Sudan I CS-MIPs at the surface of carboxyl polystyrene by core-shell emulsion polymerization with a two-step temperature-rising process. The amount of prepolymer solution, the amount of SDS, and the two-step temperature-rising polymerization were optimized to control the shell thickness of the polymers. Molecular recognition properties of CS-MIPs were systematically investigated by adsorption tests, and structural analogues were also studied for selectivity examination. The synthetic conditions and mechanism were discussed in detail, and a spherical model was constructed to evaluate the influence of shell thickness on binding capacity.

\section{Experimental}

\subsection{Reagents}

Sudan dyes (Sudan I, II, III and IV) were purchased from Shanghai Chemical Reagents Co. (Shanghai, China). Styrene, ethyleneglycol dimethacrylate (EGDMA), and methacrylic acid (MAA) were purchased from Sigma-Aldrich (Shanghai, China) and distilled under vacuum prior to use to remove inhibitor. 2,2'-Azobis(isobutyronitrile) (AIBN) was obtained from Shanghai Chemical Reagents Co. and recrystallized in methanol prior to use. Sodium dodecyl sulfate (SDS) and polyvinylpyrrolidone (PVP, MW: 24 000) were supplied by Tianjin Reagent Plant (Tianjin, China). High performance liquid chromatography (HPLC) grade acetonitrile was purchased from Merck (Darmstadt, Germany). All other chemicals and reagents were obtained from Sinopharm Chemical Reagent (Shanghai, China). All solvents and chemicals were of analytical grade and used without further purification unless otherwise specified.

\subsection{Synthesis of carboxylated polystyrene particles}

Micrometer-sized, monodisperse carboxylated polystyrene seed particles were prepared by dispersion polymerization similar to a reported procedure with necessary modification. ${ }^{21}$ In brief, PVP (1.5 g) was dissolved in ethanol (95 mL) and distilled water $(5 \mathrm{~mL})$ in a $250 \mathrm{~mL}$ three-necked, round-bottom flask. The mixture was magnetically stirred under a nitrogen atmosphere. AIBN (60 mg) in styrene $(10 \mathrm{~mL})$ and MAA $(1 \mathrm{~mL})$ were added to the above solution. After purging with nitrogen for $10 \mathrm{~min}$ at room temperature, the polymerization reaction was heated to $60{ }^{\circ} \mathrm{C}$ for $24 \mathrm{~h}$ under the nitrogen atmosphere. The obtained carboxylated polystyrene particles were purified by centrifugation and washing with ethanol and water $(1: 1, \mathrm{v} / \mathrm{v})$ three times each, and then they were dispersed in $200 \mathrm{~mL}$ distilled water.

\subsection{Preparation of CS-MIPs}

Sudan I CS-MIPs were prepared by core-shell emulsion polymerization based on the steps below. Prepolymer solutions were firstly prepared by dissolving Sudan I $(1 \mathrm{mmol})$ and MAA ( $4 \mathrm{mmol}$ ) in toluene $(4 \mathrm{~mL})$, which were stored at $4{ }^{\circ} \mathrm{C}$ in the refrigerator for $12 \mathrm{~h}$. Then, the solution of prepared carboxylated polystyrene seed particles $(20 \mathrm{~mL})$ was added to $80 \mathrm{~mL}$ of aqueous solution (containing $30 \mathrm{mg}$ SDS) in a $250 \mathrm{~mL}$ threenecked, round-bottom flask. Subsequently, 1-6 $\mathrm{mL}$ of the prepolymer solutions, 1-6 mL of EGDMA, and $80 \mathrm{mg}$ of AIBN were added, followed by purging with nitrogen for $10 \mathrm{~min}$. The twostep temperature polymerization was undertaken with magnetic stirring in a nitrogen atmosphere at $50{ }^{\circ} \mathrm{C}$ for $2 \mathrm{~h}$. The product was further aged at $60{ }^{\circ} \mathrm{C}$ for $20 \mathrm{~h}$. The polymers were then obtained by centrifugation and rinsing with anhydrous ethanol three times to wash off residue. Afterward, the polymers were washed with methanol-acetic acid solution $(9: 1, \mathrm{v} / \mathrm{v})$ in a Soxhlet extractor for $24 \mathrm{~h}$ to remove the template molecule and obtain the final Sudan I CS-MIPs. For comparison, the coreshell non-imprinted polymers (NIPs) were also prepared using the same procedures and conditions only without the addition of template molecule Sudan I.

In order to test the adsorption capacity of CS-MIPs, different amounts of prepolymer solutions were added for comparison. The MIPs prepared by adding 1, 1.5, 2, 2.5, 3 and $4 \mathrm{~mL}$ of prepolymer solution were named MIP1, MIP2, MIP3, MIP4, MIP5 and MIP6, respectively, for simplicity. It should be noted that in all the preparations, the mole ratio of MAA to EGDMA was kept at a constant value of 0.2 , and the amount of polystyrene seed particles was kept constant $(20 \mathrm{~mL})$. As a control, NIPs were prepared using identical prepolymer solutions according to the same procedures and conditions, only without the addition of template Sudan I. For example, corresponding NIP2 was given for simplicity.

\subsection{Characterization}

The morphologies of polymers were observed using a scanning electron microscope (SEM, Hitachi S-4800, Japan, operating at $20 \mathrm{kV}$ ) and a transmission electron microscope (TEM, JEM1230 , operating at $100 \mathrm{kV}$ ). Size distribution was determined by a laser particle analyzer (MASTERSIZE2000, Malvern Instruments, UK). A FT-IR spectrometer (Thermo Nicolet Corporation, USA) was employed to examine the infrared spectra of samples using a pressed $\mathrm{KBr}$ tablet method. The thermostability and purity was tested by thermogravimetry (TG) analysis using a ZRY-2P thermal analyzer (Mettler Toledo). $\mathrm{N}_{2}$ adsorptiondesorption isotherms and structure parameters were obtained via Brunauer-Emmett-Teller (BET) analysis by Full-automatic Specific Surface Instruments (3H-2000BET-A, Beishide Instruments, Beijing, China). A HPLC-UV setup (Skyray Instrument Inc., China) was used for adsorption amounts evaluation.

\subsection{Adsorption experiments}

Adsorption of Sudan I from acetonitrile solutions was carried out in batch experiments. The static adsorption test was 
performed by allowing a constant amount of CS-MIPs to reach the adsorption equilibrium with a Sudan I standard solution of known concentration. The procedure was as follows: $20 \mathrm{mg}$ of CS-MIPs were put into several $5 \mathrm{~mL}$ flasks, each containing $2.0 \mathrm{~mL}$ of Sudan I acetonitrile solutions of different concentrations. After incubation at room temperature for $12 \mathrm{~h}$, the samples were centrifuged and the supernatant solutions were collected and filtrated through a $0.45 \mu \mathrm{m}$ membrane, and the concentrations of the solutions were determined using HPLCUV. The binding amount $(Q)$ of template molecules onto MIPs could be obtained using eqn (S1), that is, by subtracting the free concentrations of template after MIPs adsorption from the initial concentrations of template molecules. The maximum binding capacity $\left(Q_{\max }\right)$ and dissociation constant $\left(K_{\mathrm{d}}\right)$ could be estimated by processing with the following Scatchard equation:

$$
\frac{Q}{C_{\mathrm{e}}}=\frac{Q_{\max }}{K_{\mathrm{d}}}-\frac{Q}{K_{\mathrm{d}}}
$$

where, $Q$ is the amount of Sudan I adsorbed onto the CS-MIPs at equilibrium, $C_{\mathrm{e}}$ is the free Sudan I concentration at equilibrium, $K_{\mathrm{d}}$ is the dissociation constant, and $Q_{\max }$ is the apparent maximum amount that can be bound. $K_{\mathrm{d}}$ and $Q_{\max }$ can be obtained from the slope and intercept of the linear curve plotted in $Q / C_{\mathrm{e}}$ versus $Q$, respectively.

Meanwhile, the dynamic adsorption test was carried out by monitoring the amount of Sudan I adsorbed by the CS-MIPs, as follows: $20 \mathrm{mg}$ of CS-MIP particles were dispersed in $2 \mathrm{~mL}$ acetonitrile solutions containing $3 \mathrm{mmol} \mathrm{L}^{-1}$ of Sudan I in $5 \mathrm{~mL}$ flasks, and then the mixtures were continuously oscillated in a thermostatically controlled water bath at room temperature for 0-150 min (i.e., 0, 10, 20, 30, 40, 50, 60, 90, 120 and $150 \mathrm{~min}$, respectively). After that, the polymers were removed centrifugally, and the supernatant solutions were collected and their concentrations determined using HPLC-UV, which were similar to those observed in the static adsorption test.

The same procedures were used to test the adsorption amounts of NIPs. Moreover, selectivity experiments were conducted by using Sudan II, III and IV that are structurally similar to Sudan I.

For the HPLC-UV procedure, a $\mathrm{C}_{18}$ column (Arcus EP-C ${ }_{18}$, $5 \mu \mathrm{m}, 4.6 \times 150 \mathrm{~mm}$ Column, Waters) was used as the analytical column. HPLC conditions optimized for the Sudan dyes were as follows: mobile phase, acetonitrile-water ( $95: 5, \mathrm{v} / \mathrm{v})$; flow rate, $1.0 \mathrm{~mL} \mathrm{~min}^{-1}$; room temperature; UV detection, $478 \mathrm{~nm}$ for Sudan I and $520 \mathrm{~nm}$ for Sudan II, III and IV; injection volume, $20 \mu \mathrm{L}$.

\section{Results and discussion}

\subsection{Preparation of CS-MIPs}

Ideal imprinted materials generally require that the templates are situated at the surface or in close proximity to the materials' surface allow the complete removal of templates, a good accessibility to the target species, and a low mass-transfer resistance. These properties are difficult to achieve in conventional bulk imprinted materials without regular shapes. In this work, emulsion polymerization was adopted. The preparation and imprinting process of the CS-MIPs is schematically illustrated in Fig. 1. In the first step, carboxyl groups were introduced to the surface of polystyrene particles by dispersion polymerization, followed by easy chemical modification using MAA for facilitating copolymerization with functional monomers. Then, the carboxyl group-capped polystyrene particles were suspended in aqueous solution with an appropriate emulsifying agent (SDS). After a two-step temperature-rising emulsion polymerization, a uniform imprinting layer was coated on the surface of the polystyrene particles. Finally, recognition sites located at the surface of the obtained MIPs were shaped after the removal of the template molecules. Compared to the complex surface modification for the general preparation of CS-MIPs, the present procedure was simple and easy to control. Herein, three kinds of strategies were successively employed to form a uniform shell. One is the two-step temperature-rising polymerization strategy. In the first polymerization stage at low temperature, polymerization proceeded slowly, resulting in a thin oligomer layer forming at the surface of the polystyrene particles, which induced the following polymerization to occur at the surface of the polystyrene. The shell was mainly shaped during the second stage at higher temperature with faster polymerization. The second strategy was easy to carry out by adjusting the amount of SDS to adjust the polymer's structure and size ranges. The third strategy was readily conducted by adjusting the amount of prepolymer solution to adjust shell thickness. Thus, by combining the three strategies, uniform core-shell structured MIPs could be attained.

\subsection{Characterization of the CS-MIPs}

The prepared CS-MIPs were characterized by SEM/TEM, laser particle analysis, BET analysis, FT-IR and TGA. SEM and TEM were employed to capture the detailed morphologies of polystyrene and CS-MIPs. As seen in Fig. 2A, the modified polystyrene particles were monodisperse with highly smooth surfaces and spherical morphology, with a uniform size of $1.5 \mu \mathrm{m}$. After polymerization, MIPs possessed a rough surface with imprinted shell layer in the surface of polystyrene, and this image revealed an average particle diameter of about $2.9 \mu \mathrm{m}$ (Fig. 2B). Fig. 2C shows the SEM image of core-shell microspheres with a relatively uniform size distribution, and the shell

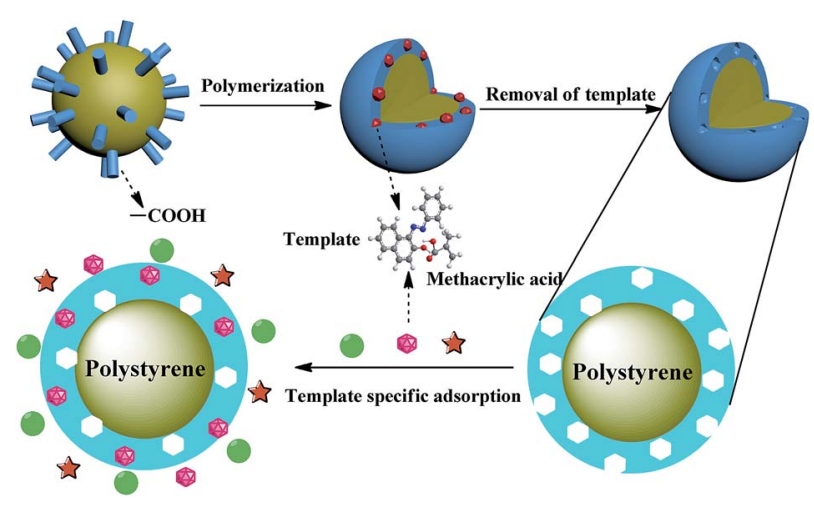

Fig. 1 Schematic illustration for preparation process of CS-MIPs. 

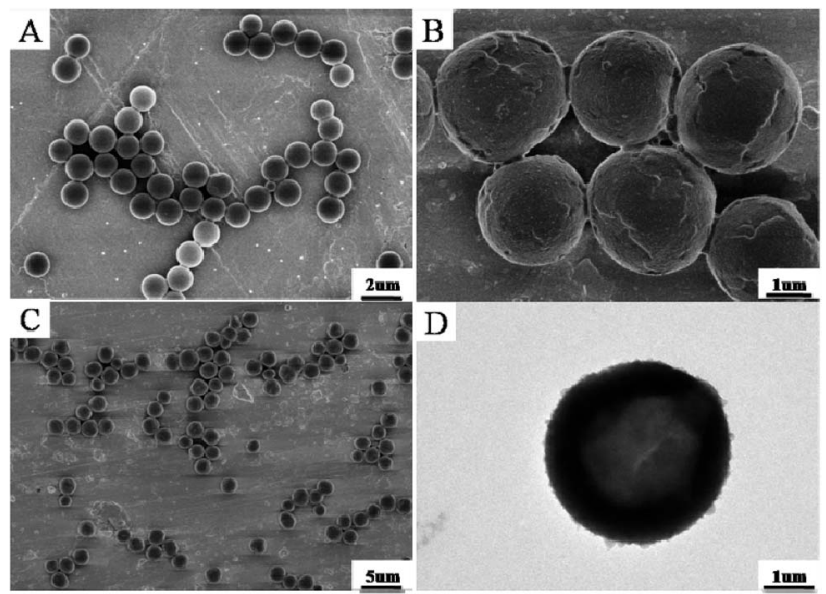

$\mathrm{D}$
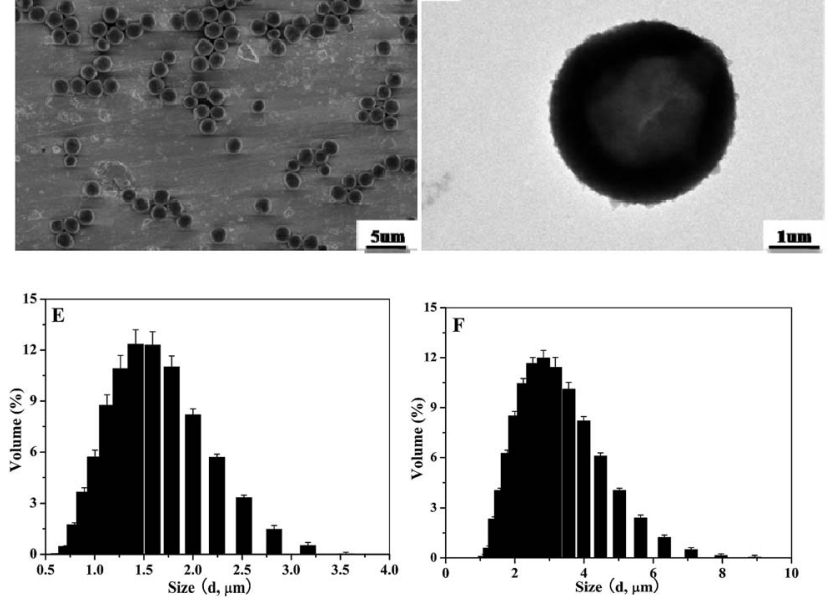

Fig. 2 SEM images of (A) modified polystyrene seeds prepared by dispersion polymerization, and (B and C) CS-MIPs prepared by coreshell emulsion polymerization. (D) TEM image of CS-MIPs prepared by core-shell emulsion polymerization. Particle size distribution of (E) modified polystyrene and (F) CS-MIPs prepared by core-shell emulsion polymerization. The CS-MIPs correspond to MIP2.

layer with a diameter of $700 \mathrm{~nm}$ could clearly be seen by TEM observation in Fig. 2D. By the virtue of the present three combined strategies, uniform CS-MIPs with different sizes could be synthesized. Moreover, the size distributions of the microparticles were obtained by laser particle analysis. As observed in Fig. 2E and F, the intensity/volume contribution versus diameters of microparticles displayed a good size distribution and a dominant distribution peak around $1.5 \mu \mathrm{m}$ for polystyrene and $3.0 \mu \mathrm{m}$ for CS-MIPs, which was allowed to be overestimated comparing with that of SEM/TEM images. For unification and simplicity, the particle size data measured by laser particle analysis was selected for further work. In addition, by BET analysis, the specific surface area of CS-MIPs was measured at $43.27 \mathrm{~m}^{2} \mathrm{~g}^{-1}$ higher than that of the corresponding NIPs $\left(22.92 \mathrm{~m}^{2} \mathrm{~g}^{-1}\right)$, as seen in Table S1. $\dagger$ Fig. S1A $\uparrow$ shows $\mathrm{N}_{2}$ adsorption-desorption isotherms of the CS-MIPs and corresponding NIPs. The average pore size of CS-MIPs and NIPs were 9.42 and $5.62 \mathrm{~nm}$, respectively (Fig. S1B $\uparrow$ ). These results proved that the binding cavities were formed on the surface of CS-MIPs by the template molecules.

Fig. 3A shows the FT-IR spectra of monodisperse carboxylated polystyrene and uniform CS-MIPs. The wide and strong absorption band at around $1730 \mathrm{~cm}^{-1}$ could be assigned to stretching vibrations of $\mathrm{C}=\mathrm{O}$ in the CS-MIPs. Two new absorption peaks at around 1262 and $1155 \mathrm{~cm}^{-1}$ also appeared, which could be attributed to the stretching vibration absorption
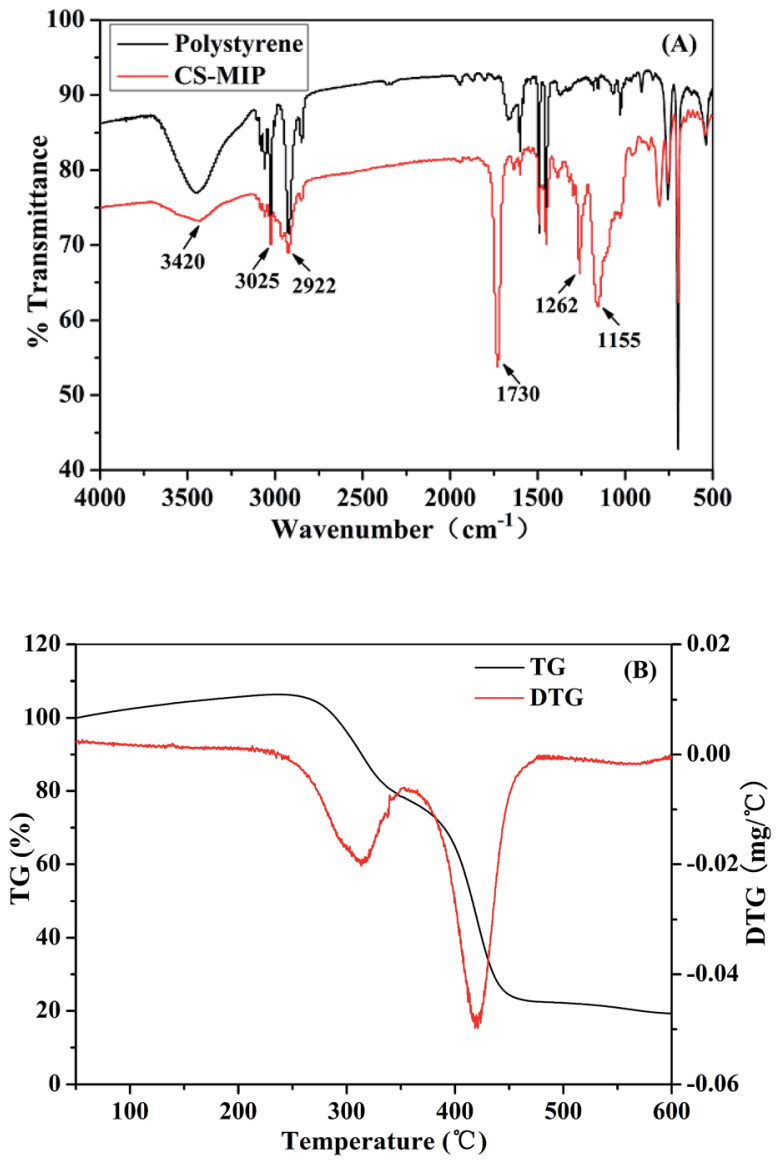

Fig. 3 (A) FT-IR spectra of polystyrene and CS-MIPs (MIP2) and (B) TG/ DTG curves of CS-MIPs (MIP2).

of saturated $\mathrm{C}-\mathrm{O}$ bonds in ester groups. This result indicated that ester groups in EGDMA had been successfully grafted to the surface of the polystyrene. Meanwhile, after polymerization, the wide and strong absorption band at around $3420 \mathrm{~cm}^{-1}$, which could be ascribed to the stretching vibration of $\mathrm{O}-\mathrm{H}$ in the carboxyl group, obviously became weak. Also, the bands at around 3025 and $2922 \mathrm{~cm}^{-1}$ became weak, possibly being stretching vibration of $\mathrm{C}-\mathrm{H}$ in the phenyl group. Therefore, CS-MIPs using polystyrene as the core were successfully prepared.

For TG analysis, a sample material is placed on an arm of a recording microbalance, which is placed in a furnace. The furnace temperature is controlled in a pre-programmed temperature/time profile, or in the rate-controlled mode, where the pre-programmed value of the weight change imposes the temperature change in the way necessary to achieve and maintain the desired weight-change rate. Fig. 3B shows the TG and DTG curves of CS-MIPs. With the temperature increasing from 50 to $240{ }^{\circ} \mathrm{C}$, it was strange that their weight did not decrease, which may be caused by two possibilities. In the first possibility, the rate of weight loss for CS-MIPs was very slow, and the CS-MIPs were very stable below $240{ }^{\circ} \mathrm{C}$. In the other possibility, the CS-MIPs presented much higher specific surface areas. The TG analysis proceeded in the nitrogen atmosphere, and the pore structure and surface of CS-MIPs could adsorb a 
small amount of nitrogen. In the range of $240-350{ }^{\circ} \mathrm{C}$, the sample loss was mainly due to the loss of polystyrene. The peak temperature of the CS-MIPs was $313{ }^{\circ} \mathrm{C}$, whereas a high rate of weight loss was presented at temperatures ranging from 350 to $470{ }^{\circ} \mathrm{C}$, and the residue amounts were $19.38 \%$. The weight loss was very likely to result from the decomposition of cross-linkers. Hence, the prepared CS-MIPs fully demonstrated good thermal stability at temperatures lower than $240{ }^{\circ} \mathrm{C}$. These observations could also be clearly explained from DTG curve.

\subsection{Influence factor examination}

It could be clearly seen that the amounts of prepolymer solution had a significant influence on the sizes of the MIP particles, from the preliminary experiments. The conventional mole ratio of the template molecules, functional monomers and crosslinking agents, $1: 4: 20$, was adopted and displayed a good imprinting effect. With the fixed proportion of the three, different volumes of the prepolymer solutions were added. As shown in Table 1, with the increase of prepolymer solution, the particle size of MIPs gradually increased. Moreover, when the amounts of polymer solution increased to $4 \mathrm{~mL}$, semi-solid form polymers were produced, which were a bit like those prepared by bulk polymerization. So, $4 \mathrm{~mL}$ of prepolymer is the boundary of emulsion polymerization and bulk polymerization. Meanwhile, the MIPs showed very poor size uniformity. When the prepolymer solution was below $3 \mathrm{~mL}$, particle size increased gradually with the increase of prepolymer solution; at the same time, the MIP particles were smoother and the consistency was better. When the prepolymer solution was $1.5 \mathrm{~mL}$, the residual and polydispersity index (PDI) were $0.619 \%$ and $0.698 \%$, respectively, which meant that the MIP particles were homogeneous. The thickness of MIP shells could further be tuned within the range of 1.3-8.5 $\mu \mathrm{m}$ by controlling the amount of prepolymer solutions. Hence, with other reaction conditions unchanged, the shell thickness was dependent on the amount of prepolymer solution. The maximum binding capacity of six different CS-MIPs template in acetonitrile was determined. As shown in Fig. S2, $\uparrow$ obviously,
MIP2 prepared by using $1.5 \mathrm{~mL}$ prepolymer solution offered the highest binding capacity. So, $1.5 \mathrm{~mL}$ of prepolymer solution was selected in the following experiments.

In core-shell emulsion polymerization, the amount of SDS has a significant effect on the sample particle size. Herein, the amount of SDS was investigated in order to control the polymer size. It can make the combination of monomer and water into a fairly stable emulsion. The appropriate amount of SDS is required. On one hand, if the amount of SDS is too little, it is difficult for the polystyrene to stably disperse in solution; on the other hand, if SDS is too much, it is easy to cause monomers to form small particles but not aggregate on the surface of the polystyrene. As seen in Table S2, $\uparrow$ the thickness of CS-MIPs (MIP2) decreased gradually with the increase of SDS. CS-MIPs using $30 \mathrm{mg}$ of SDS could maintain the formation of a grafted layer in the interface of the shell, showing satisfactory residual and consistency. The more SDS used, the smaller the CS-MIPs size was, but with the increase in SDS, CS-MIPs also easily formed micelles, being prone to forming secondary particles of cross-linking agent. Therefore, follow-up experiments were carried out by using $30 \mathrm{mg}$ of SDS.

Meanwhile, in order to ensure as much as possible that the shell layer be fabricated on the surface of polystyrene, two-step temperature-rising polymerization was performed with magnetic stirring under a nitrogen atmosphere at $50{ }^{\circ} \mathrm{C}$ for $2 \mathrm{~h}$. The resultant product was further aged at $60{ }^{\circ} \mathrm{C}$ for $20 \mathrm{~h} \cdot{ }^{22}$ At first, the temperature was set as low as possible so that the rate of polymerization reaction was slow, to ensure that the polymerization of monomers and crosslinking agents in the solution slowly occurred on the polystyrene surface to form oligomers. Once the oligomers deposited on the surface of the polystyrene, an elevated temperature was immediately used for the rapid formation of a shell.

\subsection{Model construction}

Based on the above influence examinations, we assumed that these template molecules located within a shell thickness of $x$

Table 1 Effect of prepolymer solution amount on the particle size of polystyrene and polymers ${ }^{a}$

\begin{tabular}{|c|c|c|c|c|c|c|}
\hline Polymers & Prepolymer solution (mL) & $d(0.5)^{b}$ & $D[3,2]^{c}$ & Shell thickness & Residual $^{d}$ & $\mathrm{PDI}^{e}$ \\
\hline Polystyrene & - & 1.569 & 1.535 & 0 & $6.520 \%$ & 0.227 \\
\hline MIP1 & 1 & 4.220 & 4.264 & 1.365 & $1.363 \%$ & 2.75 \\
\hline MIP3 & 2 & 9.801 & 9.057 & 3.762 & $0.682 \%$ & 3.18 \\
\hline MIP4 & 2.5 & 23.534 & 11.851 & 5.158 & $0.534 \%$ & 0.639 \\
\hline MIP5 & 3 & 29.783 & 17.461 & 7.963 & $0.753 \%$ & 0.601 \\
\hline
\end{tabular}

${ }^{a}$ The data including $d, D$, residual and PDI were obtained from the laser particle analyzer. ${ }^{b}$ The diameters of $50 \%$ particles were less than the presented value in the column. ${ }^{c}$ The average diameter of surface area of the particle, $D[3,2]=\frac{\sum d^{3}}{\sum d^{2}}$. ${ }^{d}$ Residual will be a reliable result when the value is below $1 \%$. The "residual" means the difference value between the experimentally determined value and predicted (fitted) value, which is directly obtained from the instrument. ${ }^{e}$ Polydispersity index (PDI), PDI $=D_{\mathrm{w}} / D_{\mathrm{n}}$, where, $D_{\mathrm{w}}$ and $D_{\mathrm{n}}$ mean the mass average diameter and number average diameter, respectively. Herein, PDI below 0.5 suggested a monodisperse system, ranging from 0.5 to 0.7 suggested an approximate monodisperse system, above 0.7 suggested not a monodisperse system. 
from the surface could be removed by eluting solution on the core with a fixed scale of $r$, while when $x$ exceeded a certain value, the template molecules in the interior imprinted sites within the highly cross-linked matrix could not be completely removed, which would render those binding sites invalid. The spheroidal model can be constructed as shown in Fig. 4A. The effective volume of imprinted materials that can rebind target species could be defined as:

$$
V=\frac{4}{3} \pi\left[(r+x)^{3}-r^{3}\right]
$$

In general, the $r$ value is fixed since the support particles were polymerized in the same way, which will not change with the imprinted procedure used in the next polymerization synthesis. As seen from Fig. 4B, when the $x$ value is very small, all the imprinting molecules can be cleared off within the shell thickness of $x$, and the imprinting cavities can combine with template molecules rapidly. With the increase of the $x$ value, the molecules within the particles could not be removed effectively to expose binding sites, and therefore, the binding capacity of the spherical MIP particles with identical $r$ values in the polystyrene core would be reduced (Fig. 4B). As seen, the binding capacity of MIP1 was lower than MIP2, and this phenomenon may be explained if the core of MIP1 accounted for the proportion of the particles above MIP2 and the shell of MIP1 was less than MIP2 (Table 1); however, the core of the polystyrene did not have any binding capacity. On the other hand, the binding capacity of MIP3 and MIP4 were lower than MIP2 because the template molecules at the interior of the shell
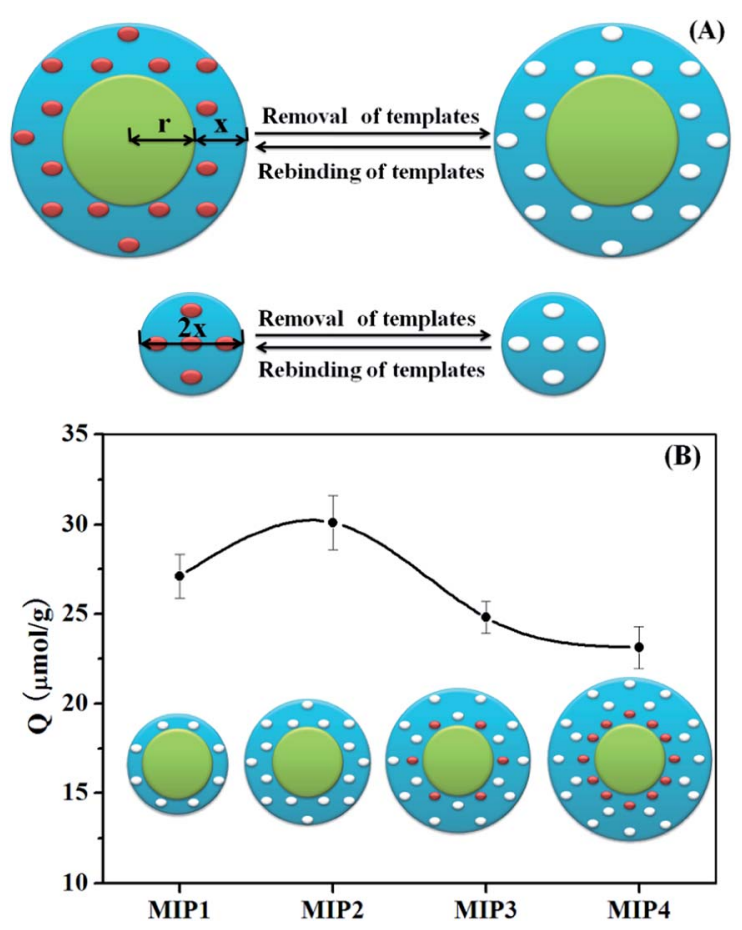

Fig. 4 (A) Schematic illustration of the constructed model of the CSMIPs. (B) Binding capacity changes of CS-MIPs with the increase in shell thickness, and the four different MIPs have identical cores. became hard to elute with the increase of shell thickness, and thus, adsorption capacity as well as the effective imprint sites were also gradually reduced. When forming uniform MIP particles less than a scale of $2 x$, all the imprinted sites can be effectively used to bind template molecules. When the CS-MIPs possessed a diameter size of $6.73 \mu \mathrm{m}$ (Table 1), and the solid core of polystyrene had a radius $r$ of $0.77 \mu \mathrm{m}$, it was roughly concluded that all the imprinted molecules could be completely removed from the polymers with a shell thickness $x$ equal to or less than $2.60 \mu \mathrm{m}$. Thus, a uniform sphere model can be further generalized with a scale diameter of $2 x$ (Fig. 4A). Template molecules situated in the spherical MIP particles with a diameter size of $5.20 \mu \mathrm{m}$, will be completely eluted and therefore the produced cavity sites will all imprint the targeted analytes. Consequently, the maximum adsorption capacity as well the maximum imprinting efficiency will be attained.

\subsection{Binding studies of the CS-MIPs}

Fig. S3A $\uparrow$ shows the binding isotherms of four different CS-MIPs and NIP2, by plotting the correlation of saturated adsorption amounts of polymers and equilibrium concentrations of Sudan I. Similar adsorption trends of MIPs were exhibited owing to their excellent monodispersity and similar core-shell structures. MIP2 showed the highest binding capacity among the MIPs, much higher than that of the corresponding NIPs (Fig. S3A $\dagger$ ) and displayed the highest imprinting factor of 2.69 (Table S3†), indicating that MIP2 possessed excellent selectivity for the template molecule. Scatchard analysis was used to assess the binding isotherms. Using the Scatchard equation, the plots for Sudan I adsorbed onto MIPs and NIPs were obtained. There were two apparent sections within the plot that can be considered as two straight lines, as shown in Fig. S4, $\uparrow$ taking MIP2 as an example. The results implied that there were two types of binding sites of high and low affinity for MIPs due to imprinting, ${ }^{23,24}$ and the rebinding/specific recognition of Sudan I was primarily dependent on hydrogen bonding. In contrast, only one straight line was observed for the NIPs (data not shown), revealing that NIPs had only low affinity binding sites from the lack of the imprinting process. ${ }^{23,24}$ It also hinted that the interaction of Sudan I with NIPs was mainly from nonspecific adsorption such as van der Waals interactions. The adsorption isotherm parameters are listed in Table $\mathrm{S} 3 \dagger$ and further confirm that MIP2 possessed the optimal molecular adsorption properties.

In addition, the ratio of the binding capacity to the effective mass ratio means the binding efficiency unit mass, and then the relationship between the binding efficiency unit mass and the core's radium ( $r$ ) of the CS-MIPs can be provided. Consequently, according to the relationship, we can deduce the critical values, which possibly results in invalid binding sites and thus decreased efficiency. Considering MIP2 as an example, the diameter is $6.733 \mu \mathrm{m}$ and radius is $3.3665 \mu \mathrm{m}$, and then the radius of the core is $0.7675 \mu \mathrm{m}$ by subtracting the shell thickness $(2.599 \mu \mathrm{m})$ from the radius $(3.3665 \mu \mathrm{m})$. Therefore, by the eqn (2), the core volume and shell volume of the CS-MIPs could be obtained as 1.894 and $157.919 \mu \mathrm{m}^{3}$, respectively. So, the ratio 
of shell volume to the total volume of the CS-MIPs is 0.988 , as shown in eqn (S2). $\uparrow$ Owing to similar density, the shell volume ratio is just equal to the mass ratio. So, the binding capacity unit mass can be attained by dividing the mass ratio by the determined binding capacity $(Q)$, that is, $30.5 \mu \mathrm{mol} \mathrm{g}{ }^{-1}$ for MIP2. The value is in good agreement with the experimentally determined

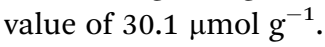

Other sorption models such as Langmuir, Freundlich and Langmuir-Freundlich were also employed to further evaluate the binding isotherms, as shown in Fig. S3B-D. $\dagger$ Related model parameters are listed in Table S4. $\dagger$ It can be observed that for all the four CS-MIPs, the Langmuir-Freundlich isotherm model yielded better fits than the Langmuir and Freundlich models, for correlation coefficients $\left(R^{2}\right)$ above 0.99 , which arises from its ability to simultaneously model both subsaturation and saturation behaviors. ${ }^{25-27}$ Additionally, MIP2 had the highest concentration of binding sites per gram of polymers $\left(N_{\mathrm{t}}=\right.$ $\left.126.16 \mu \mathrm{mol} \mathrm{g}^{-1}\right)$ and the largest median binding affinity $(\alpha=$

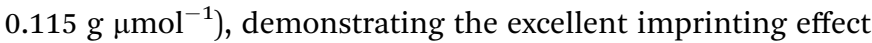
due to a number of specific binding sites on the CS-MIPs.

Dynamic binding experiments were carried out to estimate the molecular recognition properties of CS-MIPs using MIP2 as an example. As can be seen from Fig. 5A, 40 min was needed to reach adsorption equilibrium for MIP2; in the same adsorption time, the binding capacity of MIP2 was remarkably higher than that of NIP2, indicating that the uniform spherical core-shell structure of MIPs is favorable for mass transfer and binding capacity. Furthermore, the dynamic binding was investigated using different models including pseudo-first-order, pseudosecond-order, Elovich and intra-particle diffusion, ${ }^{28-30}$ as displayed in Fig. 5B, and the obtained fitting results are summarized in Table S5. $\dagger$ With the highest correlation coefficient of $R^{2}=0.9997$, a pseudo-second-order model provided the most suitable correlation for the CS-MIPs adsorption. The pseudosecond-order equation can be expressed as:

$$
\frac{t}{Q_{t}}=\frac{1}{k_{2} Q_{\mathrm{e}}{ }^{2}}+\frac{t}{Q_{\mathrm{e}}}
$$

where, $Q_{t}$ is the instantaneous adsorption amount of Sudan I in the adsorbent at time $t$, and $k_{2}$ is the adsorption rate constant. The obtained $Q_{\mathrm{e}}$ of $31 \mu \mathrm{mol} \mathrm{\textrm {g } ^ { - 1 }}$ calculated from the pseudosecond-order model agreed well with the $Q_{\mathrm{e}}$ of $30.1 \mu \mathrm{mol} \mathrm{\textrm {g } ^ { - 1 }}$ from experimental results. The curve in the entire time period proved better for predicting the kinetic process under the same experimental conditions than other models. Therefore, it can be concluded the adsorption followed a pseudo-second-order kinetics model.

The selective binding characteristic of CS-MIPs was evaluated toward three competitive Sudan dyes as structural analogues. From the data shown in Fig. S5, $\uparrow$ the following facts can be found. MIPs showed a significantly higher adsorption capacity for Sudan I than for other competitive substrates. The adsorption capacity of NIPs for the four substrates was lower than that of MIPs. The binding capacities for Sudan II were higher than the other two as its structure is more similar to that of Sudan I. All of the above facts showed that MIPs were sensitive to the presence of Sudan I and had a good selectivity
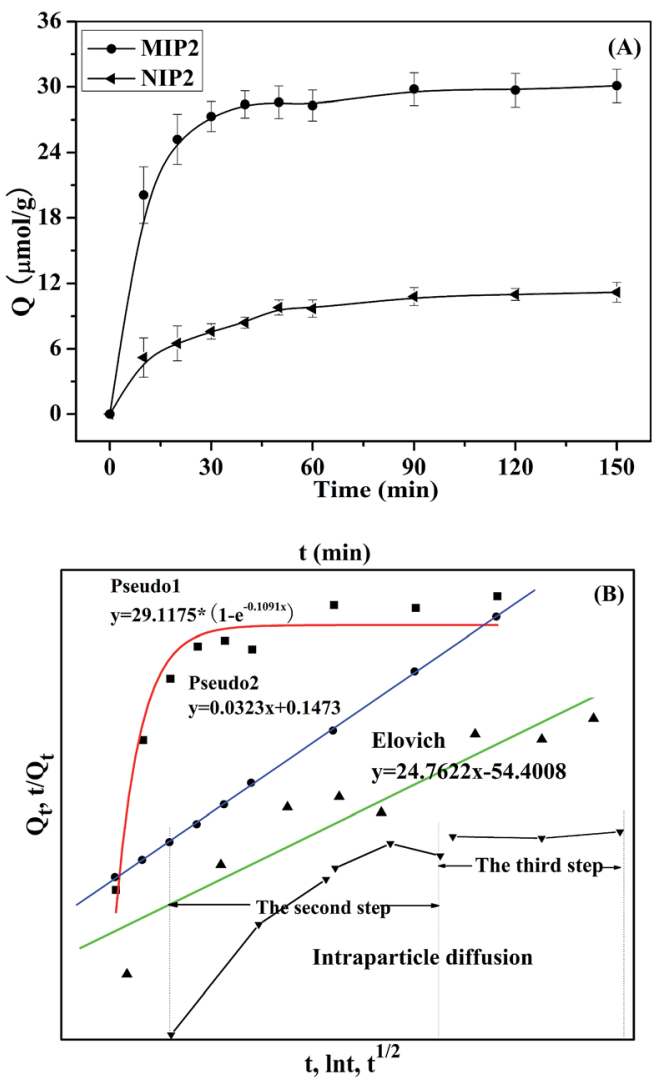

Fig. 5 (A) Kinetic uptake of MIP2 and NIP2. (B) Pseudo-first-order, pseudo-second-order, Elovich and intraparticle diffusion kinetic models of MIP2. Experimental conditions: $V=2.0 \mathrm{~mL}$; mass of polymer, $20 \mathrm{mg}$; adsorption time, $12 \mathrm{~h}$.

for recognition of the imprinted Sudan I molecules. The possible reason for MIPs recognizing the template molecule was the existence of memory cavities forming during the process of polymerization. Therefore, the molecular volume and the interaction between the target molecule and binding sites may be the two possible explanations for the selectivity of the imprinted molecule over the analogs. Obviously, the presented template played a vital role in the selectivity of molecular recognition.

\section{Conclusions}

In summary, new kinds of CS-MIP microspheres were successfully prepared by core-shell emulsion polymerization in the presence of SDS via three combined strategies and applied as models to explore the correlation between shell thickness and binding capacity. The thickness of the shell is effectively controlled by optimizing the amount of prepolymer solution, the amount of SDS and using a two-step temperature-rising polymerization. It was deduced that all the imprinted molecules could be completely removed from CS-MIPs if their shell thickness was equal to or less than $2.60 \mu \mathrm{m}$. The resultant CS-MIPs demonstrated high binding capacity and fast adsorption kinetics; the adsorption behavior was found to follow Langmuir-Freundlich isotherm and pseudo-second-order 
kinetic models. In addition, high selectivity was observed. A spherical model was constructed with a correlation between shell thickness and binding capacity. The obtained CS-MIPs had an average particle size of only a few micrometers, and thus, they could be extended for use as highly selective packing sorbents of HPLC columns.

Furthermore, the attained correlation between shell thickness and binding capacity has important guiding significance for core-shell molecular imprinting. The combination of imprinting technology and core-shell polymers opens a new window of interest to the exploration of functionalized polymers and provides new opportunities in applications involving highly selective recognition of targeted species, and therefore, the high-efficiency enrichment and removal of trace analytes from complicated matrices. The studies on structural size and mechanism can not only offer an effective way to prepare MIPs, but also greatly enrich the research of the molecular imprinting technique.

\section{Acknowledgements}

This work was financially supported by the National Natural Science Foundation of China $(21275158,21105117)$, the Innovation Projects of the Chinese Academy of Sciences (KZCX2-EW206), the 100 Talents Program of the Chinese Academy of Sciences.

\section{Notes and references}

1 L. X. Chen, S. F. Xu and J. H. Li, Chem. Soc. Rev., 2011, 40, 2922.

2 D. Cai, L. Ren, H. Z. Zhao, C. J. Xu, L. Zhang, Y. Yu, H. Z. Wang, Y. C. Lan, M. F. Roberts, J. H. Chuang, M. J. Naughton, Z. F. Ren and T. C. Chiles, Nat. Nanotechnol., 2010, 5, 597.

3 X. T. Shen, L. H. Zhu, N. Wang, L. Ye and H. Q. Tang, Chem. Commun., 2012, 48, 788.

4 Y. P. Duan, C. M. Dai, Y. L. Zhang and L. Chen, Anal. Chim. Acta, 2013, 758, 93.

5 J. H. Li, Y. Y. Wen and L. X. Chen, Chin. J. Chromatogr., 2013, 31, 181.

6 F. T. C. Moreira, R. A. F. Dutra, J. P. C. Noronha and M. G. F. Sales, Biosens. Bioelectron., 2011, 26, 4760.

7 G. Wulff, Chem. Rev., 2002, 102, 1.

8 J. Orozco, A. Cortés, G. Z. Cheng, S. Sattayasamitsathit, W. Gao, X. M. Feng, Y. F. Shen and J. Wang, J. Am. Chem. Soc., 2013, 135, 5336.
9 F. Puoci, G. Cirillo, M. Curcio, O. I. Parisi, F. Iemma and N. Picci, Expert Opin. Drug Delivery, 2011, 8, 1379.

10 A. Ribeiro, F. Veiga, D. Santos, J. J. Torres-Labandeira, A. Concheiro and C. Alvarez-Lorenzo, Biomacromolecules, 2011, 12, 701.

11 E. Saridakis and N. E. Chayen, Trends Biotechnol., 2013, 31, 515.

12 A. Bossi, F. Bonini, A. Turner and S. A. Piletsky, Biosens. Bioelectron., 2007, 22, 1131.

13 H. C. Chen, D. Y. Yuan, Y. Y. Li, M. J. Dong, Z. H. Chai, J. Kong and G. Q. Fu, Anal. Chim. Acta, 2013, 779, 82.

14 X. P. Jia, M. L. Xu, Y. Z. Wang, D. Ran, S. Yang and M. Zhang, Analyst, 2013, 138, 651.

15 L. Qin, X. W. He, W. Zhang, W. Y. Li and Y. K. Zhang, J. Chromatogr. A, 2009, 1216, 807.

16 C. J. Tan, H. G. Chua, K. H. Ker and Y. W. Tong, Anal. Chem., 2008, 80, 683.

17 G. J. Guan, R. Y. Liu, Q. S. Mei and Z. P. Zhang, Chem.-Eur. J., 2012, 18, 4692.

18 K. Yoshimatsu, K. Reimhult, A. Krozer, K. Mosbacha, K. Sode and L. Ye, Anal. Chim. Acta, 2007, 584, 112.

19 Y. Jin, M. Jiang, Y. Shi, Y. Lin, Y. Peng, K. Dai and B. Lu, Anal. Chim. Acta, 2008, 612, 105.

20 D. Gao, Z. Zhang, M. Wu, C. Xie, G. Guan and D. Wang, J. Am. Chem. Soc., 2007, 129, 7859.

21 Z. Zhang, S. F. Xu, J. H. Li, H. Xiong, H. L. Peng and L. X. Chen, J. Agric. Food Chem., 2012, 60, 180.

22 S. F. Xu, J. H. Li and L. X. Chen, J. Mater. Chem., 2011, 21, 4346.

23 H. Shaikh, N. Memon, M. I. Bhanger, S. M. Nizamani and A. Denizli, J. Chromatogr. A, 2014, 1337, 179.

24 P. Luliński and D. Maciejewska, Mater. Sci. Eng., C, 2013, 33, 1162.

25 R. J. Umpleby, S. C. Baxter, Y. Z. Chen, R. N. Shah and K. D. Shimizu, Anal. Chem., 2001, 73, 4584.

26 A. M. Rampey, R. J. Umpleby, G. T. Rushton, J. C. Iseman, R. N. Shah and K. D. Shimizu, Anal. Chem., 2004, 76, 1123.

27 C. Herdes and L. Sarkisov, Langmuir, 2009, 25, 5352.

28 J. M. Pan, L. Z. Li, H. Hang, R. R. Wu, X. H. Dai, W. D. Shi and Y. S. Yan, Langmuir, 2013, 29, 8170.

29 J. H. Zhu, S. Y. Wei, H. B. Gu, S. B. Rapole, Q. Wang, Z. P. Luo, N. Haldolaarachchige, D. P. Young and Z. H. Guo, Environ. Sci. Technol., 2012, 46, 977.

30 S. Sadasivam, K. S. Kandasamy, P. Kalaamani, S. N. Ganapathi and T. W. Kang, J. Chem. Eng. Data, 2011, 56, 4024. 